\section{CCL18 Activates Fibroblast-like Synoviocytes in Patients with Rheumatoid Arthritis}

\section{To the Editor:}

Fibroblast-like synoviocytes (FLS) proliferate in the synovial tissue of patients with rheumatoid arthritis (RA), and contribute to chronic inflammation and the destruction of articular cartilage due to the production of a variety of cytokines, chemokines, and matrix metalloproteinases (MMP) ${ }^{1}$. The expression of CC chemokine ligand 18 (CCL18) was shown to be increased in the RA synovium ${ }^{2,3}$; however, the pathogenic role of CCL18 remains unclear. In this study, we investigated the expression of a recently identified receptor for CCL18, phosphatidylinositol transfer membrane-associated phosphatidylinositol transfer protein 3 (PITPNM3) ${ }^{4}$ in the RA synovium, and the stimulatory effects of CCL18 on FLS.

Synovial tissue samples were obtained from patients with RA $(n=4)$ and osteoarthritis $(\mathrm{OA})(\mathrm{n}=4)$ undergoing joint replacement surgery. RA patients were a median 65 years old (range $48-85 \mathrm{yrs}$ ), with median disease duration 9 years (range 2.5-30 yrs) and median C-reactive protein level $1.23 \mathrm{mg} / \mathrm{dl}$ (range $0.68-2.85$ ). All RA patients were positive for rheumatoid factor and anticitrullinated protein antibodies. All subjects provided informed consent. The experimental protocol was approved in advance by the Ethics Committee of Tokyo Medical and Dental University.

CCL18-positive cells were observed in the synovial lining, sublining, and perivascular regions of the RA synovium (Figure 1A). CCL18 expression was minimal in the OA synovium (Figure 1C). Western blotting analysis showed that CCL18 expression was significantly higher in the RA synovium than in the OA synovium (Figure 1E, 1F). Double-immunofluorescence staining revealed that most CD68-positive macrophages expressed CCL18 (Figures 1G-1I), and von Willebrand factor (vWF)-positive vascular endothelial cells were also positive for CCL18 (Figures 1J-1L). These results indicated that CCL18 expression was increased in the RA synovium and macrophages were the source, which is consistent with previous studies ${ }^{2,3}$. We also found that endothelial cells expressed CCL18 in RA synovial tissue.

We then showed the expression of PITPNM3, a receptor for CCL18, in the synovial lining, sublining, and follicle-like aggregates of RA synovial tissue (Figure 1M). PITPNM3 expression was minimal in the OA synovium (Figure 1O). Increased PITPNM3 expression in the RA synovium was confirmed by Western blotting (Figure 1Q, 1R). Double-staining showed that CD68-positive macrophages and FLS (vimentin-positive fibroblast-like appearance cells) expressed PITPNM3 (Figures 1S-1X), while vWF-positive endothelial cells did not (data not shown).

The expression of PITPNM3 in in vitro cultured FLS established from RA and OA synovial tissues ${ }^{5}$ was also analyzed by Western blotting. PITPNM3 expression in RA FLS was significantly higher than that in OA FLS (Figure 2A, 2B). To analyze the pathogenic role of CCL18 in RA, we examined the stimulatory effect of CCL18 on RA FLS. FLS established from RA synovial tissue were incubated with CCL18. The production of interleukin 6 (IL-6), CCL2, and MMP-3 was significantly enhanced by stimulation with CCL18 in a dose-dependent manner (Figures 2C-2E). We also analyzed the effect of CCL18 on the proliferation of FLS. Cellular proliferation was not induced by incubation with CCL18 (Figure 2F). Incubation with CCL18 did not alter the motility of FLS as analyzed by the scrape motility assay (data not shown).

CCL18 expression was found to be increased in synovial tissue ${ }^{2,3}$, synovial fluid ${ }^{2,6}$, and serum in RA, and serum CCL18 levels correlated with disease severity ${ }^{7}$. However, the pathogenic role of CCL18 in RA has not been established, and the receptor for CCL18 has not been identified. In 2011, Chen, et al identified PITPNM3 as a functional receptor for CCL18 $8^{4}$.

In our study, we confirmed that CCL18 was expressed by RA synovial macrophages and also found that endothelial cells expressed CCL18. PITPNM3, a receptor for CCL18, was also highly expressed in the RA synovium by macrophages and FLS. CCL18 enhanced IL-6, CCL2, and
MMP-3 production in RA FLS in vitro. IL-6 has a wide range of functions on lymphocytes, hepatocytes, hematopoietic progenitor cells, and fibroblasts, and also plays important roles in autoimmune diseases. Blockade of IL-6 signaling by anti-IL-6 receptor monoclonal antibody is effective in RA. CCL2 may cause monocyte migration into the synovium ${ }^{8}$. MMP-3 is thought to contribute to pannus invasion and cartilage degradation ${ }^{9}$. Collectively, CCL18 released from synovial macrophages and endothelial cells in the RA synovium may activate FLS and is partly involved in the pathogenesis of RA. CCL18 could induce the migration of T cells, B cells, monocytes/macrophages, and dendritic cells ${ }^{10}$. Therefore, CCL18 may also influence inflammatory cell accumulation into the RA synovium.

CCL18 has been shown to induce the phosphorylation of proline-rich tyrosine kinase 2, focal adhesion kinase, and Src family kinase via PITPNM3 in cancer cells ${ }^{4}$. Further studies are needed to clarify the important signaling pathways by which CCL18 stimulation of FLS produces inflammatory mediators.

The interaction of CCL18 and PITPNM3 could play a role in the pathogenesis of RA by activating FLS in the synovium.

AIKO TAKAYASU, MSc, Department of Medicine and Rheumatology, Graduate School of Medical and Dental Sciences, and Section of Microbiology and Immunology, Graduate School of Health Care Sciences, Tokyo Medical and Dental University; YOSHISHIGE MIYABE, MD; WAKA YOKOYAMA, MD; KAYOKO KANEKO, MD, PhD; SHIN FUKUDA, MD; NOBUYUKI MIYASAKA, MD, PhD, Department of Medicine and Rheumatology, Graduate School of Medical and Dental Sciences, Tokyo Medical and Dental University; CHIE MIYABE, MD, Department of Medicine and Rheumatology, Graduate School of Medical and Dental Sciences, Tokyo Medical and Dental University, and Department of Dermatology, Tokyo Medical University; TETSUO KUBOTA, MD, PhD, Section of Microbiology and Immunology, Graduate School of Health Care Sciences, Tokyo Medical and Dental University; TOSHIHIRO NANKI, MD, PhD, Department of Medicine and Rheumatology, and Department of Pharmacovigilance, Graduate School of Medical and Dental Sciences, Tokyo Medical and Dental University, Tokyo, Japan.

Address correspondence to Dr. T. Nanki, Department of Medicine and Rheumatology, Graduate School of Medical and Dental Sciences, Tokyo Medical and Dental University, 1-5-45, Yushima, Bunkyo-ku, Tokyo 113-8519, Japan. E-mail: nanki.rheu@tmd.ac.jp

Supported in part by Grants-in-Aid for Scientific Research from the Ministry of Health, Labor, and Welfare, Japan, and by the Japanese Ministry of Education, Global Center of Excellence (GCOE) Program, International Research Center for Molecular Science in Tooth and Bone Diseases.

\section{REFERENCES}

1. Muller-Ladner U, Ospelt C, Gay S, Distler O, Pap T. Cells of the synovium in rheumatoid arthritis. Synovial fibroblasts. Arthritis Res Ther 2007;9:223

2. Schutyser E, Struyf S, Wuyts A, Put W, Geboes K, Grillet B, et al. Selective induction of CCL18/PARC by staphylococcal enterotoxins in mononuclear cells and enhanced levels in septic and rheumatoid arthritis. Eur J Immunol 2001;31:3755-62.

3. Radstake TR, van der Voort R, ten Brummelhuis M, de Waal Malefijt M, Looman M, Figdor CG, et al. Increased expression of CCL18, CCL19, and CCL17 by dendritic cells from patients with rheumatoid arthritis, and regulation by Fc gamma receptors. Ann Rheum Dis 2005;64:359-67.

4. Chen J, Yao Y, Gong C, Yu F, Su S, Chen J, et al. CCL18 from tumor-associated macrophages promotes breast cancer metastasis via PITPNM3. Cancer Cell 2011;19:541-55.

5. Nanki T, Nagasaka K, Hayashida K, Saita Y, Miyasaka N. Chemokines regulate IL- 6 and IL-8 production by fibroblast-like synoviocytes from patients with rheumatoid arthritis. J Immunol 


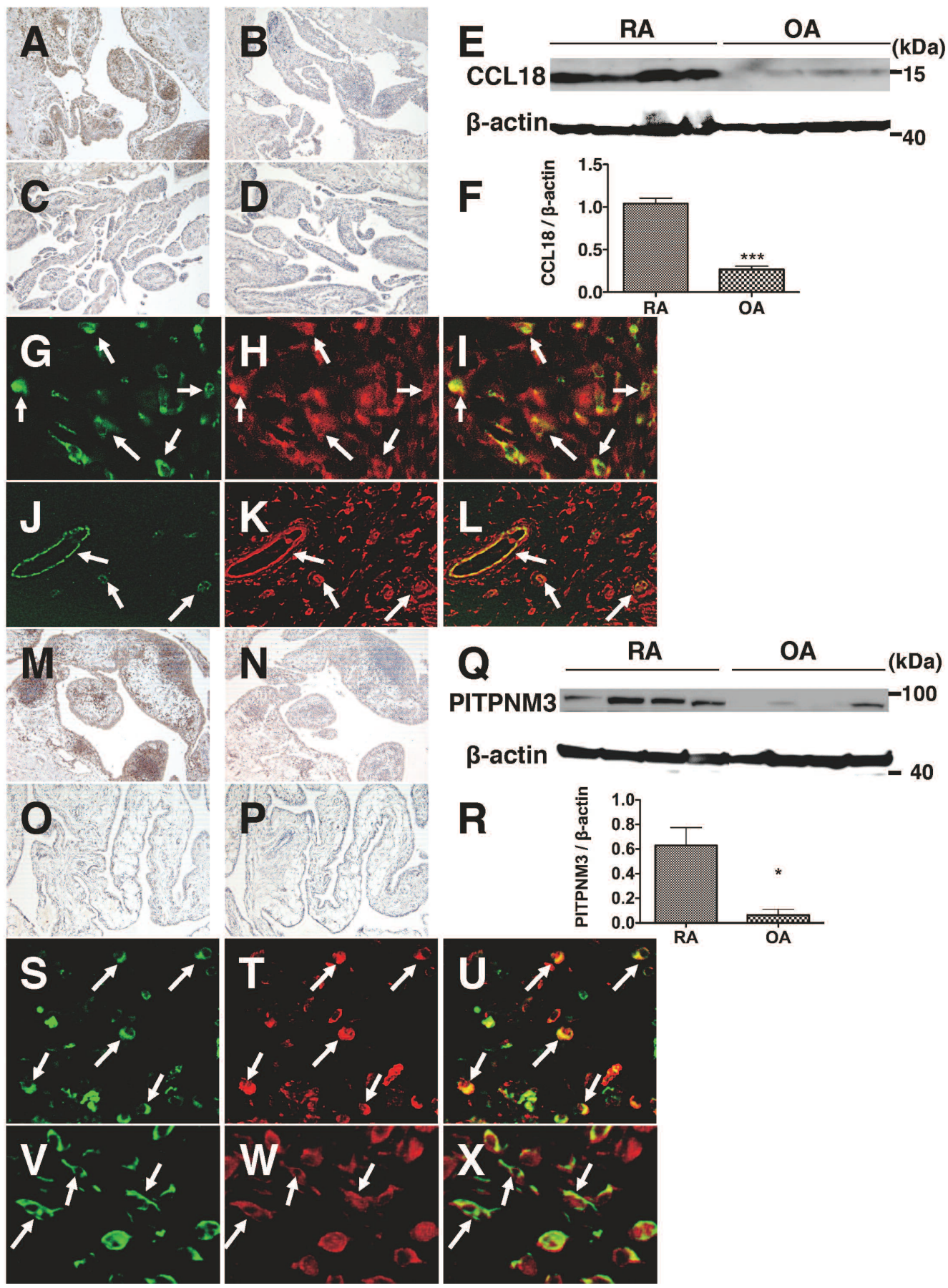

Figure 1. Expression of CCL18 and PITPNM3 in the RA synovium. Synovial tissues were obtained from 4 RA (A, B) and 4 OA patients (C, D), and CCL18 expression was examined by immunohistochemistry. Samples were stained with anti-CCL18 antibody (LifeSpan BioSciences; A, C) or isotype-matched control antibody $(B, D)$. Original magnification $\times 100$. CCL18 expression in RA and OA synovial tissues was also analyzed by Western blotting with anti-CCL18 antibody and anti- $\beta$-actin monoclonal antibody (Cell Signaling Technology; E). Protein expression of CCL18 relative to $\beta$-actin in RA and OA is shown $(\mathrm{F})$. Values are mean \pm SEM. ${ }^{* * *}$ p $<0.001$, Student $\mathrm{t}$ test. Sections of RA synovial tissues were double-stained with anti-CD68 (KP1; DakoCytomation) or -vWF (F8/86; DakoCytomation) monoclonal antibody and anti-CCL18 antibody, and were analyzed by fluorescence microscopy as follows: CD68 (G), CCL18 (H), merged image (I) of (G) and (H), vWF (J), CCL18 (K), merged image (L) of (J) and (K). Arrows indicate double-positive cells. Original magnification $\times 400$ in G to I, $\times 200$ in J to L. Expression of PITPNM3 in RA (M and N) and OA $(\mathrm{O}$ and P) synovial tissues was examined by immunohistochemistry. Samples were stained with anti-PITPNM3 antibody (GeneTex; M and O) or isotype-matched control antibody (N and P). Original magnification $\times 100$. PITPNM3 expression in RA and OA synovial tissues was determined by Western blotting (Q). Relative expression of PITPNM3 to $\beta$-actin in RA and OA is shown (R). Values are mean \pm SEM. * $p<0.05$, Student $t$ test. RA synovial tissues were double-stained with anti-CD68 or vimentin (V9; DakoCytomation) monoclonal antibody, and anti-PITPNM3 antibody. CD68 (S), PITPNM3 (T), merged image (U) of (S) and (T), vimentin (V), PITPNM3 (W), merged image $(\mathrm{X})$ of $(\mathrm{V})$ and $(\mathrm{W})$. Arrows indicate double-positive cells. Original magnification $\times 400$. 


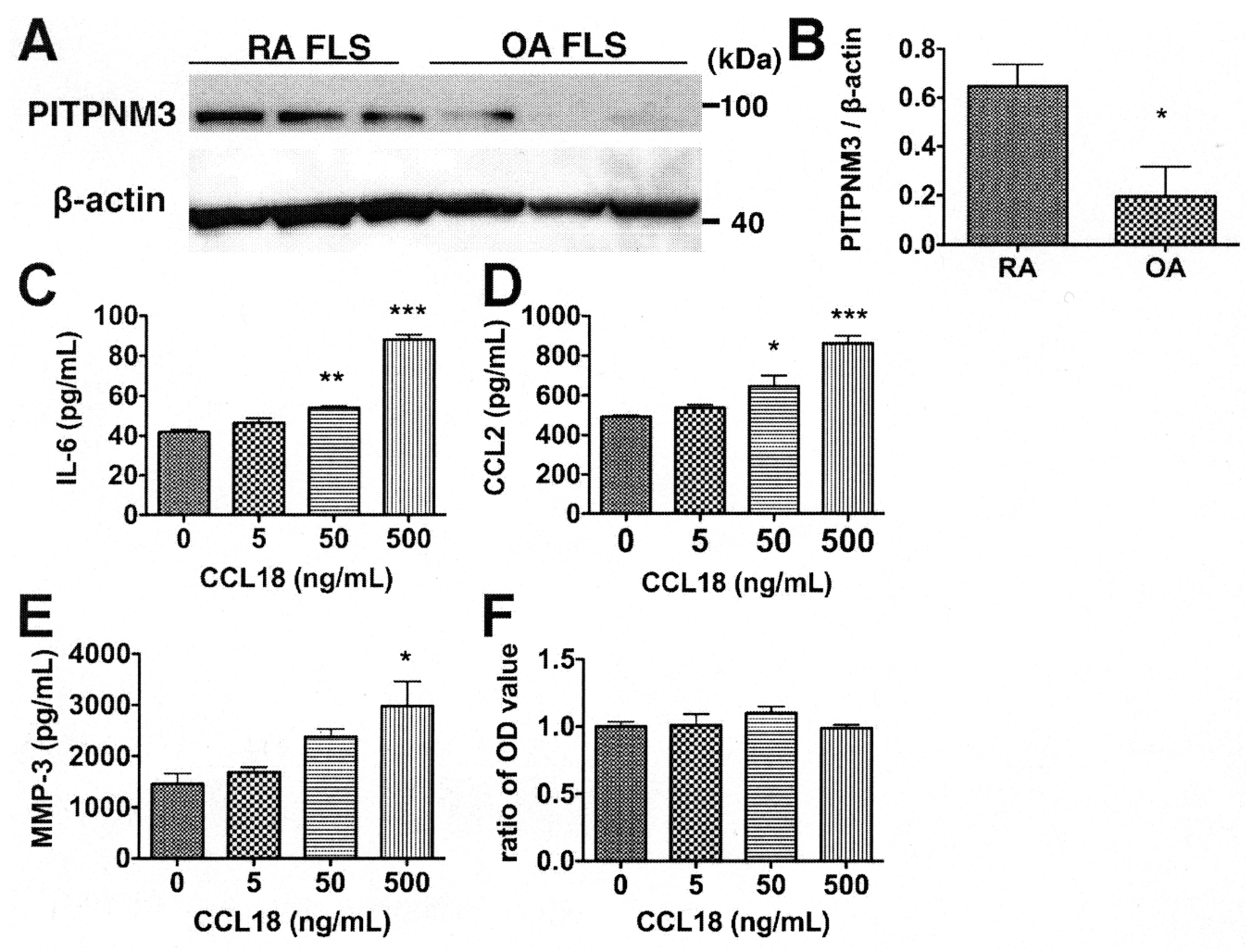

Figure 2. Expression of PITPNM3 in cultured FLS and the stimulatory effect of CCL18. Expression of PITPNM3 in in vitro cultured FLS from RA and OA synovial tissues was determined by Western blotting (A). Relative expression of PITPNM3 to $\beta$-actin in RA FLS and OA FLS is shown (B). Values are mean \pm SEM. $* \mathrm{p}<0.05$, Student $\mathrm{t}$ test. FLS $(2 \times$ $10^{3}$ cells/well) from RA synovial tissue were cultured overnight in a 96-well plate in Dulbecco's modified Eagle's medium (DMEM) with 10\% fetal bovine serum. Then medium was replaced with serum-free DMEM followed by addition of the indicated concentration of CCL18 (R\&D Systems). After 72 hours, IL-6, CCL2, and MMP-3 concentrations in culture supernatant were measured by ELISA (R\&D systems) (C: IL-6; D: CCL2; E: MMP-3), and cell proliferation was examined using a cell counting kit (Cell Counting Kit-8; Dojindo; F). Data are presented as means \pm SEM of 1 of 3 independent experiments analyzed in triplicate. ${ }^{*} \mathrm{p}<0.05$; ** $\mathrm{p}<0.01$; *** $\mathrm{p}<0.005$ (one-way ANOVA with Dunnett's multiple comparison test).

2001;167:5381-5.

6. van der Voort R, Kramer M, Lindhout E, Torensma R, Eleveld D, van Lieshout AW, et al. Novel monoclonal antibodies detect elevated levels of the chemokine CCL18/DC-CK1 in serum and body fluids in pathological conditions. J Leukoc Biol 2005; 77:739-47.

7. van Lieshout AW, Fransen J, Flendrie M, Eijsbouts AM, van den Hoogen FH, van Riel PL, et al. Circulating levels of the chemokine CCL18 but not CXCL16 are elevated and correlate with disease activity in rheumatoid arthritis. Ann Rheum Dis 2007;66:1334-8.

8. Hayashida K, Nanki T, Girschick H, Yavuz S, Ochi T, Lipsky PE. Synovial stromal cells from rheumatoid arthritis patients attract monocytes by producing MCP-1 and IL- 8 . Arthritis Res 2001;3:118-26.

9. Ainola MM, Mandelin JA, Liljestrom MP, Li TF, Hukkanen MV, Konttinen YT. Pannus invasion and cartilage degradation in rheumatoid arthritis: Involvement of MMP-3 and interleukin-1 beta. Clin Exp Rheumatol 2005;23:644-50.

10. Schutyser E, Richmond A, Van Damme J. Involvement of CC chemokine ligand 18 (CCL18) in normal and pathological processes. J Leukoc Biol 2005;78:14-26.

J Rheumatol 2013;40:6; doi:10.3899/jrheum.121412 